\title{
RESONANCE RADIATION OF SODIUM VAPOR EXCITED BY ONE OF THE D LINES.
}

By R. W. WOOd ANd Fred L. MOHLer.

\section{INTRODUCTION.}

$\mathrm{T}^{\mathrm{H}}$

$\mathrm{HE}$ purpose of this investigation was to study the resonance spectrum of sodium vapor when the resonance was excited by a single D line. In a paper on "Resonance Radiation of Sodium Vapor" published by one of us in I905, it was shown that if a bulb containing pure sodium vapor was illuminated by light from a sodium flame, the vapor emitted a yellow light which spectroscopic analysis showed to be identical with the exciting light, in other words, the two $\mathrm{D}$ lines. It was suggested at this time that it would be interesting to see if both $\mathrm{D}$ lines appeared when the vapor was illuminated by a single $\mathrm{D}$ line. This would determine whether the mechanisms that give rise to the $\mathrm{D}$ lines are separate or in some way connected.

In I9I4 this experiment was tried by Wood and Dunoyer. ${ }^{2}$ This experiment was made possible by the discovery of a polarization method of separating close spectral doublets that eliminated the great loss of light involved in a high-power monochromator, but even with this method the resonance light is very faint. The spectrum of resonance excited by $D_{2}$ was photographed with exposures varying from io to I 5 hours. The majority of the plates showed only the $\mathrm{D}_{2}$ line, but owing to under exposure $D_{1}$ would not have been visible if it was less than one fourth of the intensity of $D_{2}$. Some plates indeed showed both $D_{1}$ and $D_{2}$, but the presence of $D_{1}$ in the exciting light was judged to be the cause of this. Imperfections in the optical system made it impossible to entirely remove $D_{1}$ from the exciting light and as it was sensitive to temperature changes the nature of the transmitted light sometimes changed during an exposure. It was concluded that sodium resonance excited by $D_{2}$ consisted of $D_{2}$ alone, but the results admit of two other possibilities. $D_{1}$ light may always be present though much fainter than $\mathrm{D}_{2}$, or it may only appear under certain conditions.

Other resonance phenomena show that the radiation centers in sodium are not entirely independent. One of us $^{3}$ showed in 1905 that excitation

${ }^{1}$ R. W. Wood, Phil. Mag. (6), 1o, 5r3, I905.

${ }^{2}$ Wood and Dunoyer, Phil. Mag. (6), 27 , Ior 8, I9I4.

${ }^{3}$ Wood, Phil. Mag. (6), I0, 408, I905. 
of sodium vapor by blue-green light, in the region of the band spectrum, gives rise to the $\mathrm{D}$ lines, or, at least, to a band in that region.

Strutt ${ }^{1}$ in 1915 found that resonance radiation consisting of the D lines could be excited by the 3300 doublet of sodium, the second doublet in the principal series of which the D lines are the first. When only one line of the 3300 doublet was excited by a coincident zinc line both the $D$ lines again appeared. This remarkable discovery, indicating clearly some connection between the emission centers of the doublets of the principal series of sodium, made a further study of the excitation of resonance by one of the $\mathrm{D}$ lines seem desirable. In view of results which will be mentioned further along, it may be well to point out that Strutt's results may have been due to the presence of hydrogen in his bulb of sodium vapor.

The arrangement of the apparatus and the method used in the present work differ only in minor details from the method of Wood and Dunoyer.

The chief requirements for the investigation are:

I. A method of completely separating $\mathrm{D}_{1}$ and $\mathrm{D}_{2}$ in the exciting light with the least possible reduction in the intensity of the light.

2. The preparation of bulbs containing sodium vapor that will give brilliant resonance during a prolonged heating.

3. The analysis of the light by a spectroscope giving the greatest possible intensity of light commensurate with the dispersion necessary to clearly resolve the $\mathrm{D}$ lines.

The Method of Separating the D Lines.

The polarization method of separating close spectrum lines was described by one of $\mathrm{us}^{2}$ in I9I4. Briefly the method is as follows: If plane-polarized monochromatic light is passed through a doubly refracting crystal with its direction of vibration making an angle of $45^{\circ}$ with the optic axis of the crystal, it will in general emerge elliptically polarized, but for certain thicknesses of the crystal it will be plane polarized either in the same direction as the incident light or at $90^{\circ}$ to this direction. If, now, we have light of two wave-lengths in the incident beam, the emerging beam will be in two different states of polarization due to the difference in refractive index for the two wave-lengths. It is possible to find a thickness of the crystal such that the emergent light consists of two monochromatic beams plane polarized at right angles to each other. By the use of an analyzing nicol either wave-length may be cut out and monochromatic light secured.

${ }^{1}$ R. J. Strutt, Proc. of Royal Soc., Series $A$, 9I, p. 5 II.

${ }^{2}$ Wood, Phil. Mag. (6), 27, 524, I9I4. 
To cut out one of the D lines with quartz a plate about $32 \mathrm{~mm}$. thick is required. The thickness can vary considerably for the precise optical length of path required can be secured by tilting the plate. To secure intense illumination a large plate of quartz must be used in parallel light and the faces must be optically plane, or nearly so.

Since half of the light is cut out if it is polarized by a nicol, large double-image prisms were used to polarize and analyze the light. They were placed so that only two images of the source were formed. When the quartz block was placed between them and properly oriented three images appeared, the central image consisting of two superposed images containing only $\mathrm{D}_{2}$ light and two lateral ones containing only $\mathrm{D}_{1}$ light. A slight tilting of the quartz plate changed the central image to $D_{1}$ and the lateral ones to $\mathrm{D}_{2}$.

The large quartz block measuring $85 \times 60 \times 32 \mathrm{mms}$., prepared for the experiment of Wood and Dunoyer, was refigured and supported rigidly in a brass frame arranged to rotate on an axis parallel to the optic axis of the crystal, and inclined at an angle of $45^{\circ}$ to the vertical. It was placed between two large Iceland-spar prisms of about the same size as the block of quartz and mounted with their edges (optic axes) vertical. The lenses of a large Dunoyer condenser made the light passing through the prisms parallel, and brought it to a focus on the bulb of sodium vapor.

This optical system was enclosed in a wooden box, which was kept at a constant temperature to within $0 . \mathrm{I}^{\circ} \mathrm{C}$. by a benzene thermostat. This precaution is necessary for a change in temperature of a degree or two will completely change the nature of the light transmitted by the quartz block. A long handle fastened to the supporting rod of the quartz block made it possible for an observer at the spectroscope to turn the block and adjust the apparatus for the extinction of one of the $\mathrm{D}$ lines.

\section{The Spectroscope.}

For analysis of the resonance radiation a large two-prism spectroscope furnished with portrait objectives of 3 inch aperture and 24 inch focus, loaned by the psychology department of this university, was found to give the best results. With this instrument brilliant illumination and clear resolution of the $\mathrm{D}$ lines were secured with a fairly wide slit, though the definition was not perfect. This spectroscope had been arranged for use as a monochromator, with the second slit mounted on a screw, so that it could be moved along the spectrum. For the present work the photographic plates were simply clamped against the second slit mounting. This offered a very convenient method of taking a series of exposures on the same plate, side by side instead of one above the other, as in the ordinary plateholder. 


\section{Light SOURCE.}

The source of light was a Meker burner surrounded by a chimney provided with a rectangular aperture measuring about $2 \times 5 \mathrm{cms}$. The image of this rectangle, formed by the polarizing separator, was thrown on the bulb of sodium vapor. A disk of asbestos soaked in salt solution touched the edge of the flame and this disk was revolved once in twelve hours by the hour hand gear of a clock. This device kept the sodium flame at about the proper intensity to give the maximum brilliancy of resonance. It is very important, however, to have the disk graze the flame on the side furthest removed from the lens, as by this arrangement reversal of the $\mathrm{D}$ lines is obviated. This is of fundamental importance since the resonance radiation is excited by the core of the line only.

\section{Heating Device.}

The bulb containing the sodium was supported above an asbestos chimney about two feet high and five inches in diameter, below which was placed a nest of Bunsen burners. The bulb was supported by a wire frame in such a way that it could be turned about a vertical axis, and a firmly supported pin point touched the front surface to detect any possible displacement when the bulb was rotated.

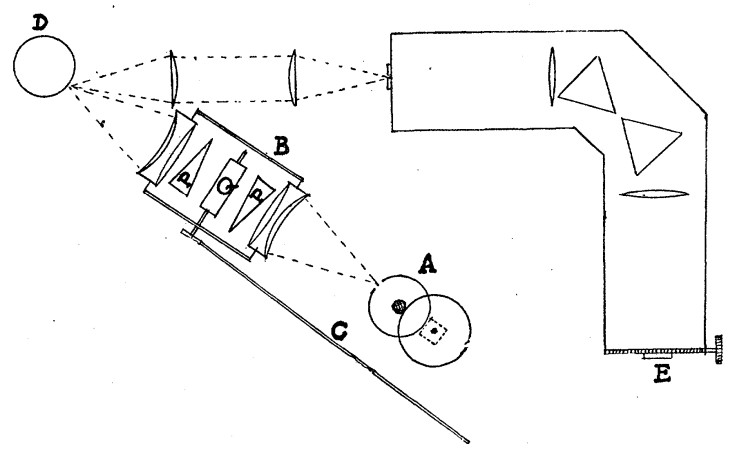

Fig. 1.

Arrangement of Apparatus.

Fig. I shows a plan of the apparatus. The light source asbestos disk and surrounding chimney is at $A . B$ is the optical system for separating the $\mathrm{D}$ lines with the long handle $C$ to turn the quartz block $Q$ between the spar prisms $P P$. The bulb of sodium vapor is at $D$, placed in the position shown so as to prevent directly reflected light from falling on the slit of the spectroscope. At $E$ is the plateholder of the spectroscope mounted on a horizontal screw. 
The improvements over the apparatus previously used are in the device for separating the $\mathrm{D}$ lines and in the spectroscope. The spectroscope gave better illumination and the system for separating the $D$ lines gave almost perfect extinction of $D_{1}$, though the extinction of $D_{2}$ was not quite so good, as $D_{2}$ has double the intensity of $D_{1}$ in the case of the comparatively feeble flame used for the excitation.

\section{Preparation of the Bulbs.}

The method used in preparing the sodium bulbs is practically that previously described by Dunoyer and Wood. ${ }^{1}$ A bulb about $5 \mathrm{~cm}$. in diameter is made as shown in Fig. 2. A piece of sodium, weighing about .2 of a gram, is put in the tube at the left, the tube immediately sealed at $A$ and the bulb connected to the pump and exhausted. The bulb is heated for about half an hour to free the glass from occluded water and the sodium is then distilled into it and

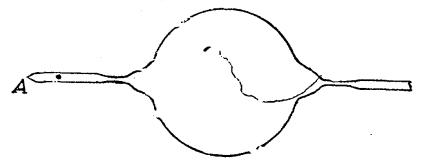

Fig. 2. the side tube sealed off. The sodium is then distilled from one side of the bulb to the other many times by heating opposite sides alternately with a Bunsen burner, while the pump is kept running and the pressure read from time to time on a McLeod gauge.

The preliminary heating prevents or at least retards the reaction of the sodium with the glass which at temperatures above $200^{\circ}$ reduces the silicon oxide and makes the glass brown and finally opaque. Bulbs of Pyrex glass, which proved to be far superior to ordinary glass in this respect, prepared in the way described, showed scarcely any color after twelve hours' heating at $220^{\circ}$, and were quite transparent, though brown, after heating twelve hours at $300^{\circ}$.

The repeated distillation of the sodium was to drive off the hydrogen which is occluded by it in large quantities. If, after distilling the sodium into the bulb the pump was cut off and the sodium driven from one side of the bulb to the other two or three times the pressure gauge indicated a rise of about $.3 \mathrm{~mm}$., and repeated distillation with the pump maintaining a vacuum of about $.002 \mathrm{~mm}$., only removed this hydrogen very slowly. The sodium vapor seemed to carry most of the hydrogen with it as it was distilled from side to side of the bulb, for when the pump was stopped and the bulb heated the pressure always increased several hundredths of a millimeter. In the preparation of one bulb the sodium was distilled back and forth across the bulb I70 times in a high vacuum after which the pump was stopped and .or $\mathrm{mm}$. of gas was

${ }^{1}$ Dunoyer and Wood, Phil. Mag. (6), 27, I027, I9I4. 
given off by the sodium when it was heated. In cases in which it was desirable to have some hydrogen present the pump was cut off as soon as the sodium distilled into the bulb. The bulb was then heated, the pressure measured, and the bulb sealed off. To test whether prolonged heating increased the amount of gas some bulbs were opened under mercury after they had been used, but the amount of gas present was not noticeably different.

The careful removal of all the hydrogen is not necessary to secure brilliant resonance, but it does affect the character of the resonance spectrum, as will be explained later.

\section{Procedure.}

To facilitate the adjustment of the apparatus for extinction of one of the $\mathrm{D}$ lines a patch of magnesium oxide was put on the surface of the bulb by burning magnesium wire below it and then removing all the oxide except a small rectangular strip. To adjust the apparatus the bulb is placed in position and turned until the light from the sodium flame falls on the patch of oxide. As magnesium oxide is a nearly perfect reflector this gives a source of light bright enough to make all adjustments. To photograph the resonance spectrum the bulb is turned slightly till the exciting light falls on clean glass. Care must be taken that no light is directly reflected into the spectroscope.

Owing to the path difference through the quartz block of rays coming from different parts of the rectangular aperture, the illumination is not strictly monochromatic $\left(D_{2}\right)$ over the entire image of the aperture. Experiments showed that we have pure $\mathrm{D}_{2}$ radiation along slightly curved and nearly vertical strips two or three millimeters in width, the distribution of the illumination being somewhat as shown in Fig. 3 .

The upper and lower portions of the image of

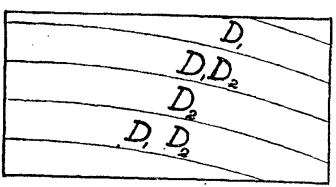

Fig. 3. the patch of resonance radiation thrown on the slit were excited by both lines when the central portion was excited by one only. For this reason any motion of the image on the slit either during the exposure, or in turning the bulb before the exposure, had to be guarded against. As in some cases only a small part of the line was single, it was necessary to compare corresponding parts of the lines of the exciting light and of the resonance light. This comparison was facilitated by the possibility of making several exposures on the same plate with the lines side by side.

The usual procedure was to first photograph the exciting light reflected from the magnesium oxide, then move the plate, turn the bulb and 
expose to the resonance light, and at the end of the exposure again turn the bulb and move the plate and expose to the exciting light.

The exposures for the resonance spectrum varied from three to fifteen hours; usually twelve hours. The exposures for the diffusely reflected exciting light, to give the same intensity as the resonance light in I2 hours, were from fifteen to thirty minutes when the same type of flame was used. The brightest resonance is secured when the flame is quite faint. Wratten and Wainwright panchromatic plates were used.

The method of estimating the intensity ratio of the $\mathrm{D}$ lines, when both appeared, was to match the two lines with sodium lines on a comparison plate made by taking a series of exposures of varying length with a sodium flame of constant intensity. The intensity ratio was assumed equal to the ratio of exposure times of lines that matched.

\section{Results.}

Most of the plates taken were of resonance excited by $\mathrm{D}_{2}$, for as $\mathrm{D}_{2}$ is about twice as bright as $D_{1}$, there are obvious advantages in trying it first. The efficiency of the polarization method of cutting out $\mathrm{D}_{1}$ was tested and it was estimated that under the best conditions $\mathrm{D}_{2}$ was at least 50 times as bright as $\mathrm{D}_{1}$, though overlapping due to irradiation of the $\mathrm{D}_{2}$ line made it impossible to be sure of the ratio.

The results of many exposures to resonance excited by $\mathrm{D}_{2}$ showed visible traces of $D_{1}$ in nearly every case, but with an intensity ratio of $D_{2}$ to $D_{1}$ that varied from about 6 to $I$, to about 20 to I. This result led at first to the suspicion that stray sodium light was in some way thrown on the spectroscope slit. All possible precautions against this source of error were taken.

When with these precautions both $\mathrm{D}$ lines appeared in the resonance spectrum a further precaution was taken to be sure the effect was not false. A narrow horizontal strip of magnesium oxide was placed so as to intercept the rectangle of resonance light in such a way that part of the resulting spectrum line was formed by resonance, and part by reflected light. Since the resonance light is much fainter than the light reflected from a white surface the comparison strip was made a dark gray by first coating the bulb with smoke, and then depositing magnesium oxide until the reflected light was of about the same intensity as that of the resonance. Fig. $4 A$ shows the form of the oxide patch,

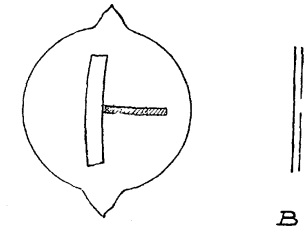

Fig. 4. the large rectangular strip being the same as that before mentioned, and the narrow shaded strip the part that intercepted the patch of resonance. 
Physical Review, Second Series, Vol. XI, January, I9 8 .
Plate I.

To face page 77 .
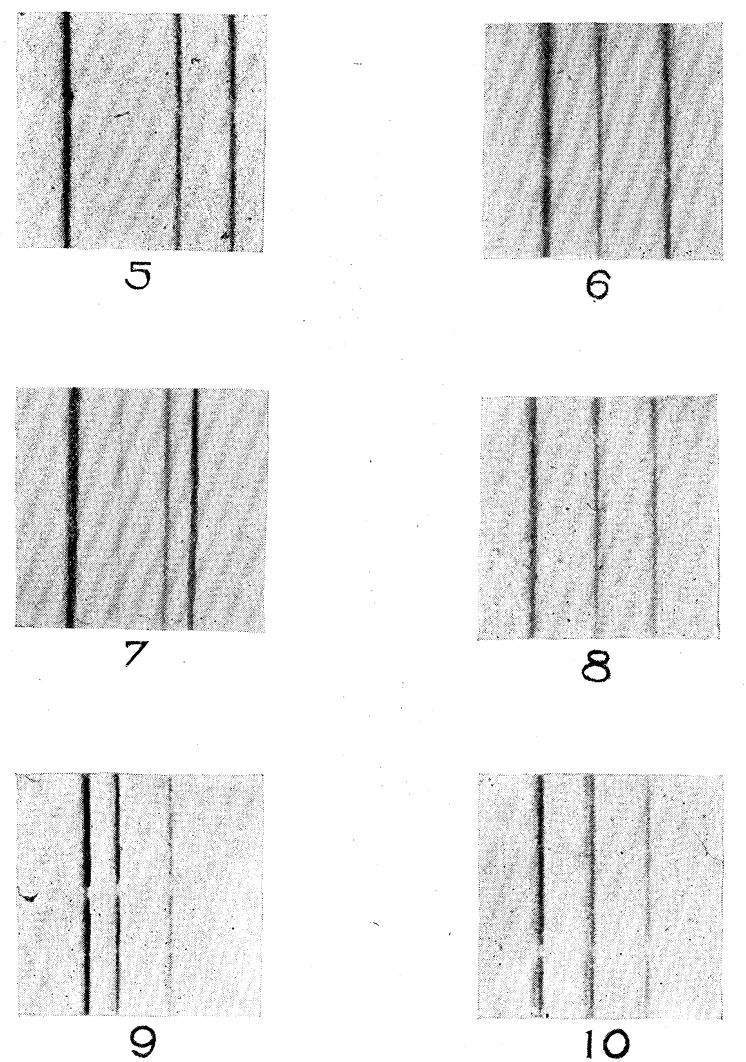

R. W. WOOD AND FRED L. MOHLER. 
Fig. $4 B$ shows the appearance of the resulting spectrum line as it appeared when this method was used. Both $D$ lines appear except at the place where the exciting light is reflected from the gray strip into the spectroscope, and there only one line is recorded. If the appearance of both lines was due to reflection of stray light from the surface of the glass there would be no break in the line. This effect was found both with resonance excited by $D_{2}$ and by $D_{1}$, and seemed to be conclusive evidence that $\mathrm{D}_{2}$ light did excite a trace of $\mathrm{D}_{1}$ light in the resonance radiation. Having verified the results by this method the gray strip was dispensed with in the later work, as it somewhat complicated the adjustment of the bulb. On all plates, however, three exposures were taken, as is shown in the accompanying plate. The plates have been enlarged about ten times. In each case the middle line, or pair of lines, is the resonance spectrum, and the two lines on each side are due to the exciting light diffusely reflected from the patch of magnesium oxide. False effects due to any change in the exciting light can thus be detected.

Comparison of a number of plates taken under different conditions of temperature, with bulbs prepared in different ways, did not at first show clearly under what conditions $D_{1}$ appeared in the resonance spectrum excited by $\mathrm{D}_{2}$. This was due to the fact that two causes contributed to the effect. However all the bulbs from which the hydrogen was not carefully removed showed $\mathrm{D}_{1}$ distinctly. Now the resonance spectrum of iodine vapor excited by the green mercury line is changed in the presence of electro-positive gases such as helium and hydrogen, the effect of the gases being to transfer energy from the radiation centers directly excited by the mercury line to other radiation centers. The effect will be described later. The possibility of a similar effect in the case of sodium resonance led to the following experiments: The effect of a change in the density of pure sodium vapor on the resonance excited by $\mathrm{D}_{2}$ was first investigated. A bulb containing sodium that was as free as possible from hydrogen was used for three exposures to resonance excited by $\mathrm{D}_{2}$ at temperatures of $210^{\circ}, 270^{\circ}$ and $340^{\circ}$ keeping all other conditions constant. The exposure at $210^{\circ}$ showed no trace of $\mathrm{D}_{1}$ in the resonance spectrum, while at $270^{\circ}$ a distinct trace of $D_{1}$ was seen. At $340^{\circ}$ the intensity ratio of $D_{2}$ to $D_{1}$ was about the same as that in a faint flame, about 2 to I, but the plate was much under-exposed and the result therefore was subject to error. Figs. 5 and 6 of the accompanying plate show the resonance of pure sodium vapor excited by $\mathrm{D}_{2}$ at $210^{\circ}$ and $300^{\circ}$ respectively.

It may be well to mention here the change in general appearance of the resonance as the temperature is raised. Resonance light becomes 
visible at about $120^{\circ}$, and appears as a faint glow throughout the bulb. As the temperature is raised the light becomes brighter at the front surface and fades out in the interior of the bulb until, finally, the light is limited to the surface and exhibits a sharp image of the source when it is focused on the bulb. At $200^{\circ}$ the resonance appears only at the surface though the image of the source is still a little indistinct, but above $250^{\circ}$ the image is as sharp as if the light was reflected from a piece of smooth paper.

The change in the resonance spectrum when hydrogen was put into the bulb was more marked than the change when the vapor pressure of the sodium increased. The resonance excited by $\mathrm{D}_{2}$ in a bulb containing $.25 \mathrm{~mm}$. of hydrogen showed $\mathrm{D}_{1}$ about a quarter as bright as $\mathrm{D}_{2}$ at $210^{\circ}$ and at $300^{\circ} \mathrm{D}_{1}$ was a third as bright as $\mathrm{D}_{2}$. Figs. 7 and 8 were taken under these conditions. The faint line in Fig. 7 is of no importance.

A similar series of exposures was taken of the resonance excited by $D_{1}$. It is difficult in this case to avoid traces of $\mathrm{D}_{2}$ in the exciting light for reasons before mentioned, and the intensity of the resonance is reduced to about half. The effect of increasing the vapor pressure or putting hydrogen in the bulb is the same in this case as with $\mathrm{D}_{2}$ excitation, though the intensity ratio of $D_{2}$ to $D_{1}$ with $D_{1}$ excitation is greater than that of $D_{1}$ to $D_{2}$ with $D_{2}$ excitation when other conditions are the same. Thus with pure sodium at $210^{\circ}$ a trace of $\mathrm{D}_{2}$ was visible (Fig. 9) while with .I $\mathrm{mm}$. of hydrogen in the bulb $\mathrm{D}_{2}$ is half as bright as $\mathrm{D}_{1}$ (Fig. Io). Some plates, where more hydrogen was in the sodium bulb, showed $\mathrm{D}_{2}$ nearly as bright as $D_{1}$ but the plates were underexposed and there was a possibility that the effect was false.

All the results mentioned above were verified by repetition of the experiments. In all about 50 plates were taken in which the resonance lines were distinct, and the other conditions favorable as far as could be ascertained.

Estimates of the intensity ratio of the $\mathrm{D}$ lines were made in each case. They agreed roughly under apparently similar conditions of vacuum, temperature of bulb, etc., but there was quite a range of uncertainty both in the estimation of the intensity ratio, and in the ability to get conditions identical in two cases. The observations may be summarized as follows:

\section{$\mathrm{D}_{2}$ Excitation.}

Bulb as free from hydrogen as possible.

At $210^{\circ}$ (no trace of $D_{1}$ ) intensity ratio of $D_{2}$ to $D_{1}$ at least 20 to $\mathrm{I}$. Fig. 5 . 
At $300^{\circ}$, ratio of $D_{2}$ to $D_{1} 5$ to I. Fig. 6 .

Bulb containing about .25 mm. of hydrogen.

At $220^{\circ}$, ratio of $D_{2}$ to $D_{1} 4$ to I. Fig. 7 .

At $300^{\circ}$, ratio of $D_{2}$ to $D_{1} 3$ to $I$. Fig. 8 .

\section{1 Excitation.}

Bulb free from hydrogen.

At $220^{\circ}$, a trace of $\mathrm{D}_{2}$ seen. Fig. 5 .

At $300^{\circ}$, ratio of $D_{1}$ to $D_{2} 3$ to $I$.

Bulb containing .I mm. of hydrogen.

At $220^{\circ}$, ratio of $D_{1}$ to $D_{2} 2$ to I. Fig. 6 .

Bulb containing $.25 \mathrm{~mm}$. of hydrogen.

At $250^{\circ}$, ratio of $D_{1}$ to $D_{2}$ possibly 3 to 2 .

\section{CONCLUSION.}

As it has been shown that the presence of hydrogen causes both $\mathrm{D}$ lines to appear when resonance is excited by one D line only, it is safe to conclude that the appearance of both $\mathrm{D}$ lines at high temperatures is due to the increase of the pressure of the sodium vapor. From the measurements of vapor tension made by Hackspill ${ }^{1}$ we can estimate the pressure of sodium vapor at the temperatures used. Extrapolating the vapor-tension temperature curve given by him gives the following values of vapor pressure:

$$
\begin{aligned}
& \text { At } 200^{\circ}, .003 \mathrm{~mm} \text {. } \\
& \text { At } 250^{\circ} \text {, .0I } \mathrm{mm} \text {. } \\
& \text { At } 300^{\circ}, .025 \mathrm{~mm} \text {. }
\end{aligned}
$$

Thus at $200^{\circ}$ the vacuum is nearly as good as in a cold bulb, but at $300^{\circ}$ the amount of sodium vapor is comparable to the amount of hydrogen present, in the bulbs made to show the effect of that gas.

There is a striking analogy to this effect of hydrogen and sodium vapor on the resonance spectrum of sodium, in the case of the resonance spectrum of iodine vapor excited by the green mercury line when traces of a chemically inert gas are present. This effect was described by one of us in I9II. ${ }^{2}$ Iodine vapor at room temperature in a high vacuum when excited by the green line of the Cooper-Hewitt mercury arc emits a spectrum consisting of a series of doublets spaced at nearly equal frequency intervals. The first member is in coincidence with the exciting line and the last (or 28th) is at wave-length 7683. If a long exposure is

1 Hackspill, Annales de Chemie et de Physique, 28, 680, I9r3.

2 Wood and J. Franck, Phil. Mag. (6), 21, p. 265. 
given it is found that traces appear of very regular bands, similar in appearance to that of the $\mathrm{A}$ line of the solar spectrum. If helium at $3 \mathrm{~mm}$. pressure is introduced into the bulb, the doublets weaken and the bands increase in intensity. As the helium pressure increases the doublets become fainter and the bands stronger in proportion; that is, there is a transfer of energy from the system or systems giving rise to the doublets, to that responsible for the band spectrum. The same thing occurs with xenon or krypton, or any other electro-positive gas. An electro-negative gas, however, merely decreases the intensity of the resonance, and so far as is now known, does not give rise to the transfer of energy. It is of course possible or even probable that there is some transfer in this case, but the intensity is so greatly reduced that its detection is difficult.

We conclude that the transfer of energy from the $\mathrm{D}_{2}$ to the $\mathrm{D}_{1}$ emission centers, or vice versa, is in some way the result of molecular collision, either of sodium with hydrogen or of sodium with sodium. It has been shown that hydrogen and sodium vapor, both electropositive, cause this transfer of energy, and the analogy to the similar transfer in the case of iodine resonance is of considerable interest.

In a bulb of pure sodium at $220^{\circ}$ the surrounding vapor is not dense enough to have an observable effect on the radiation centers, and only one line appears in the resonance spectrum. The appearance of the other line results from an increase in the collision frequency, which increase can be caused either by the introduction of hydrogen at low pressure or by increasing the density of the sodium vapor.

JOHNS Hopkins UNIVERSITY,

$$
\text { June, I9r } 7 \text {. }
$$



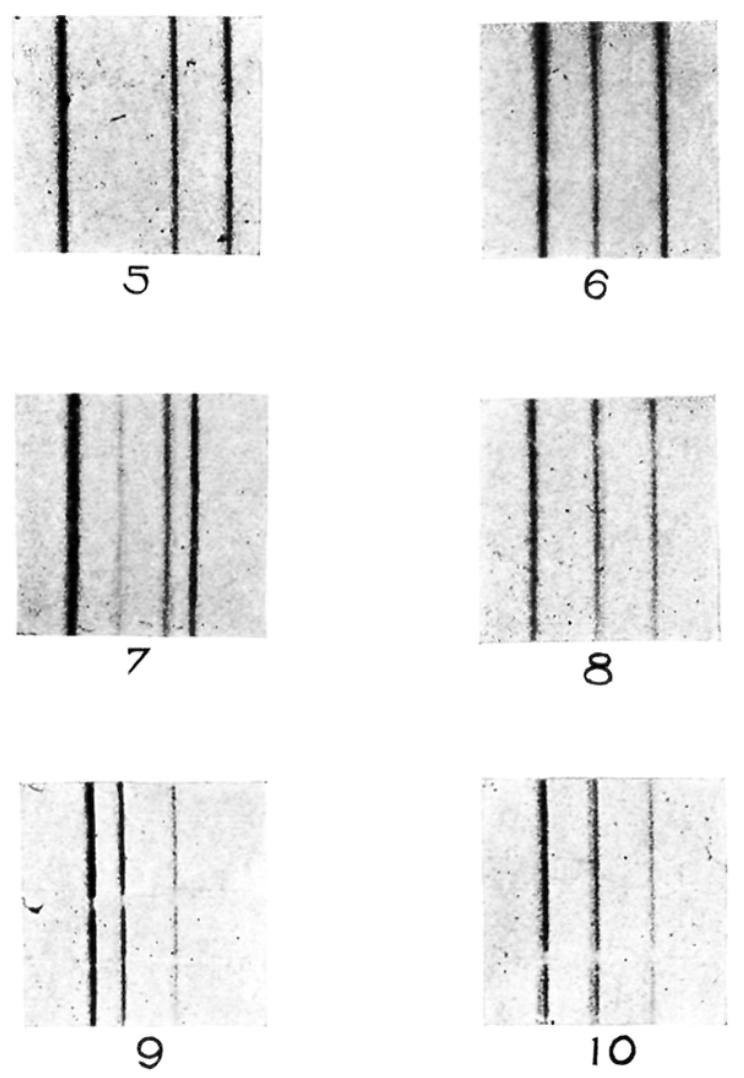